
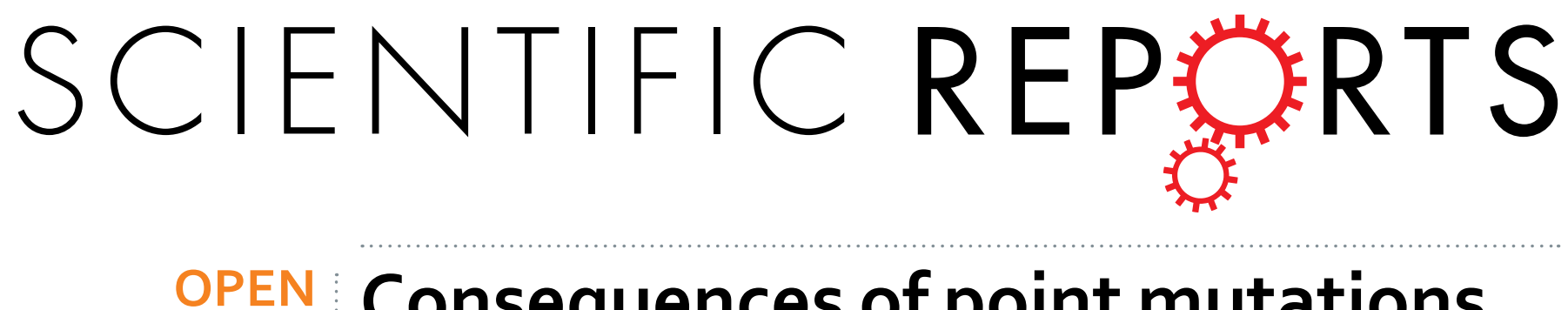

\title{
Consequences of point mutations in melanoma-associated antigen 4 (MAGE-A4) protein: Insights from \\ structural and biophysical studies
}

Received: 01 February 2016

Accepted: 12 April 2016

Published: 28 April 2016
Yoshio Hagiwara ${ }^{1}$, Lina Sieverling ${ }^{1}$, Farina Hanif ${ }^{1}$, Jensy Anton ${ }^{1}$, Eleanor R. Dickinson ${ }^{2}$, Tam T. T. Bui ${ }^{3}$, Antonina Andreeva ${ }^{4}$, Perdita E. Barran ${ }^{2}$, Ernesto Cota ${ }^{5}$ \& Penka V. Nikolova ${ }^{1}$

The Melanoma-Associated Antigen A4 (MAGE-A4) protein is a target for cancer therapy. The function of this protein is not well understood. We report the first comprehensive study on key cancer-associated MAGE-A4 mutations and provide analysis on the consequences of these mutations on the structure, folding and stability of the protein. Based on Nuclear Magnetic Resonance and Circular Dichroism, these mutations had no significant effects on the structure and the folding of the protein. Some mutations affected the thermal stability of the protein remarkably. Native mass spectrometry of wild-type MAGE-A4 showed a broad charge state distribution suggestive of a structurally dynamic protein. Significant intensity was found in relatively low charge states, indicative of a predominantly globular form and some population in more extended states. The latter is supported by lon Mobility measurements. The MAGE-A4 mutants exhibited similar features. These novel molecular insights shed further light on better understanding of these proteins, which are implicated in a wide range of human cancers.

Cancer/Testis (CT) antigens are a large family of proteins typically expressed in germ cells. CT antigens can also be overexpressed in an aberrant manner in various types of tumors such as melanoma, sarcoma, lung cancer, prostate cancer, breast cancer, ovarian cancer and a range of various other cancers ${ }^{1-5}$.

The first discovered CT antigen family member was melanoma-associated antigen A1 (MAGE-A1), which was identified as an antigen recognised by cytotoxic T lymphocytes in melanoma patients ${ }^{6}$, and was successfully cloned later ${ }^{7,8}$. Since then, extensive chromosomal sequencing identified more than 50 genes of the MAGE family ${ }^{9-18}$. Based on their expression pattern, the MAGE family can be divided into two subfamilies: type-I and type-II ${ }^{19}$. While the expression of type-I subfamily is restricted to germ line and cancer cells, type-II subfamily is expressed in normal somatic tissues. Type-I MAGEs can be subdivided into MAGE-A, -B and -C groups. MAGE-A contains 12 genes (MAGE-A1 to -A12) where MAGE-A7 is a pseudogene ${ }^{9}$. MAGE proteins consist of nearly 100 amino acid residues long $\mathrm{N}$-terminal region, followed by two tandem winged helix domains with each wing termed as WH-A and $\mathrm{WH}-\mathrm{B}$, respectively ${ }^{20}$. The $\mathrm{N}$-terminal is rich in disorder promoting residues such as Ser, Pro, Glu and Arg, and thus this region is predicted to be disordered. The C-terminal region that spans over the two winged helix domains is highly conserved amongst type I and type II MAGEs and is known as MAGE Homology Domain (MHD). The crystal structure of MHD has been determined in a free state (MAGE-A4, PDB ID: 2AW0; MAGE-A3, PDB ID: 4V0P) and in complex with NSE1 (MAGE-G1, PDB ID: 3NW0) ${ }^{20}$.

The biological functions of MAGE proteins remain poorly understood. However, many reports correlate over-expression of type-I MAGEs with cancer malignance, tumor growth and poor patient prognosis. For example, MAGE-A2 was reported to promote tumor growth in normal oral keratinocytes and inhibits cell cycle arrest

${ }^{1}$ King's College London, Faculty of Life Sciences \& Medicine, Institute of Pharmaceutical Science, Franklin-Wilkins Building, 150 Stamford St, London, SE1 9NH, UK. ${ }^{2}$ Michael Barber Centre for Collaborative Mass Spectrometry, Manchester Institute of Biotechnology, The University of Manchester, 131 Princess Street, Manchester, M1 7DN, UK. ${ }^{3}$ Biomolecular Spectroscopy Centre, King's College London, The Wolfson Wing, Hodgkin Building, London SE1 1UL. ${ }^{4}$ MRC-LMB, Francis Crick Avenue, Cambridge, CB2 00H. ${ }^{5}$ mperial College London, Faculty of Natural Sciences, London, SW7 2AZ. Correspondence and requests for materials should be addressed to P.V.N. (email: penka. nikolova@kcl.ac.uk) 
through down-regulation of p53 targets ${ }^{21}$. MAGE-A4 was also shown to abrogate p53-dependent growth arrest and apoptosis in normal oral keratinocyte ${ }^{22}$. The mechanism(s) of action of MAGE-A proteins that can lead to tumor growth have not yet been fully elucidated at a molecular level. However, MAGE-A2 was reported to interact with the DNA binding domain of p53 and to suppress p53 trans-activation by recruiting histone deacetylase $(\mathrm{HDAC})^{23}$ or by inhibiting the interaction between p53 and DNA through steric hindrance ${ }^{24}$. Most recently, MAGE-A antigens were identified as being causal contributors in the development of tamoxifen-resistant breast cancers $^{25}$. Specifically, MAGE-A2 protein was reported to localize to the nucleus and to form complexes with p53 and ER alpha, resulting in repression of the p53 pathway while increasing the ER dependent signalling. This report showed that in tamoxifen-treated breast cancer patients, there was a significant link between MAGE expression and reduced overall survival. In addition, various MAGE proteins such as MAGE-A2, -A3 and -A6, have been reported to interact with E3 ubiquitin ligase such as TRIM28, enhancing their ubiquitin ligase activity and reducing 553 protein level through a proteasome-dependent pathway ${ }^{20}$.

In contrast, tumor suppressor activity was also reported for MAGE-A4. MAGE-A4 was found to interact with the liver oncoprotein gankyrin, suppressing its tumorigenic activity ${ }^{26}$. A truncated form of MAGE-A4 (the C-terminal 107 amino acids) was reported to induce apoptosis by interacting with POZ domain/zinc finger transcription factor Miz-1 ${ }^{27}$.

To date, there are 104 MAGE-A4 DNA mutations documented in the Catalogue of Somatic Mutations in Cancer (COSMIC) database (http://cancer.sanger.ac.uk/cancergenome/projects/cosmic/). Among these mutations, 76 are missense mutations, 52 of which are located within the MHD. In addition, Caballero et al. ${ }^{28}$ identified ten missense mutations in melanoma patients and two missense mutations in tumor samples (breast cancer cell line and glioma ${ }^{28}$.

In this study, we have selected nine MAGE-A4 mutations to investigate the effects of these mutations on the structure, folding and stability of MAGE-A4, all of which are reported by Caballero et al ${ }^{28}$. Eight of these mutations are located within the MHD (E138K, P149S, G153D, E221K, E224K, E242K, P267S and R269C) and one near the C-terminus (G316R). The overall criteria for the selection of these mutations was that they mapped within the MHD of MAGE-A4, which is the only domain that had known X-ray structure. Our results reveal that although the mutations have marginal effects on the structure of MAGE-A4, some of them can affect significantly the thermal stability of the protein. In addition, both WT and mutant MAGE-A4 contain a large disordered or unfolded region as well as a structured hydrophobic core. Native mass-spectrometry (MS) shows that all of the MAGE-A4 proteins are present primarily in a monomeric form with a low abundant fraction of dimeric species. Ion mobility mass spectrometry (IM-MS) shows the WT MAGE-A4 to present in multiple conformers, whilst mutant MAGE-A4 proteins show significant compaction in the gas-phase. This study provides novel information for one of the MAGE-A proteins, namely, MAGE-A4 with regard to its biophysical properties. We have performed structure-based homology modelling, mass-spectrometry (MS), circular dichroism (CD) and nuclear magnetic resonance (NMR) to provide comprehensive analyses of the MAGE-A4 protein and its key cancer associated mutants. The finding could be exploited for cancer-based drug design and/or to inform further cancer therapies.

\section{Results}

Assessing the folding of the MAGE-A4 proteins using Far-UV CD at different temperatures. In order to assess the folding state and secondary structure of MAGE-A4 and examine the effects of mutations on them, the proteins were subjected to CD in the Far-UV region $(260-195 \mathrm{~nm})$. The locations of these selected mutations in the amino acid sequence and the crystal structure of MAGE-A4 MHD are shown in Figs 1 and 2, respectively. The spectra were recorded at $6,20,37$ and $90^{\circ} \mathrm{C}$, followed by cooling down to $37^{\circ} \mathrm{C}$ and $20^{\circ} \mathrm{C}$ respectively. The results are presented in Fig. 3. The CD spectra of each protein at 6,20 and $37^{\circ} \mathrm{C}$ were essentially identical, indicating that the WT and mutant MAGE-A4 proteins maintain their native folded state at physiological temperature. In addition, no significant difference between the WT and the mutants was observed in the spectra at each temperature, suggesting little effects of these mutations on the secondary structure. When the temperature was raised to $90^{\circ} \mathrm{C}$, all of the proteins were thermally denatured. When the temperature was cooled down to 37 and $20^{\circ} \mathrm{C}$, the spectra still clearly differed from those obtained at 6,20 and $37^{\circ} \mathrm{C}$, demonstrating that the proteins did not recover their native folded state. This indicates that the thermal unfolding of MAGE-A4 proteins is irreversible. We employed the K2D3 programme (http://k2d3.ogic.ca/) ${ }^{29}$ to estimate the secondary structure elements of WT MAGEA- 4 at physiological temperature (i.e. $37^{\circ} \mathrm{C}$ ), and this was estimated to be $29.9 \% \alpha$-helix and $17.1 \% \beta$-sheet.

The effects of mutations on the thermal stability of MAGE-A4. We employed differential scanning fluorimetry (DSF) to determine the apparent melting temperature $\left(T_{\mathrm{m}}\right)$ of MAGE-A4 proteins and assess the effects of the mutations on the thermal stability of the protein. The melting curves of MAGE-A4 proteins are shown in Fig. 4. All of the curves have the classic sigmoidal shape showing two different phenomena: unfolding and aggregation. The unfolding process of each protein occurs in the range of $20^{\circ} \mathrm{C}$. The apparent $T_{\mathrm{m}}$ of WT MAGE-A4 is $\sim 49.3^{\circ} \mathrm{C}$. The least thermally stable protein was E242K with the $T_{\mathrm{m}} \sim 43.6^{\circ} \mathrm{C}$, followed by P267S $\left(T_{\mathrm{m}}\right.$ $\left.\sim 44.6^{\circ} \mathrm{C}\right)$, E138K $\left(T_{\mathrm{m}} \sim 44.7^{\circ} \mathrm{C}\right), \mathrm{R} 269 \mathrm{C}\left(T_{\mathrm{m}} \sim 46.2^{\circ} \mathrm{C}\right)$ and P149S $\left(T_{\mathrm{m}} \sim 47.3^{\circ} \mathrm{C}\right)$. The difference between the $T_{\mathrm{m}}$ of E242K and that of WT, $\left(\Delta T_{\mathrm{m}}\right)$, was $-5.7^{\circ} \mathrm{C}$, and thus significant. Nearly $10 \%$ fraction of E242K mutant unfolds at physiological temperature. The most stable mutant was E221K, showing $T_{\mathrm{m}} \sim 54.6^{\circ} \mathrm{C}$ and $\Delta T_{\mathrm{m}}$ of $5.3^{\circ} \mathrm{C}$. The difference in the $T_{\mathrm{m}}$ between the most stable mutant $(\mathrm{E} 221 \mathrm{~K})$ and that of the least stable mutant $(\mathrm{E} 242 \mathrm{~K})$ was remarkable $\left(11^{\circ} \mathrm{C}\right)$. Other mutants $(\mathrm{G} 153 \mathrm{D}, \mathrm{E} 224 \mathrm{~K}$ and $\mathrm{G} 316 \mathrm{R})$ showed similar thermal stability to the WT with relatively similar $T_{\mathrm{m}}$, within $\sim 1^{\circ} \mathrm{C}$ difference compared to the WT. These results demonstrate that some mutations can dramatically enhance (such as E221K) or reduce (such as E242K) the thermal stability of the protein. Interestingly, the mutations that showed significant difference in the thermal stability of the proteins (E242K and E221K) affect solvent-exposed residues. 


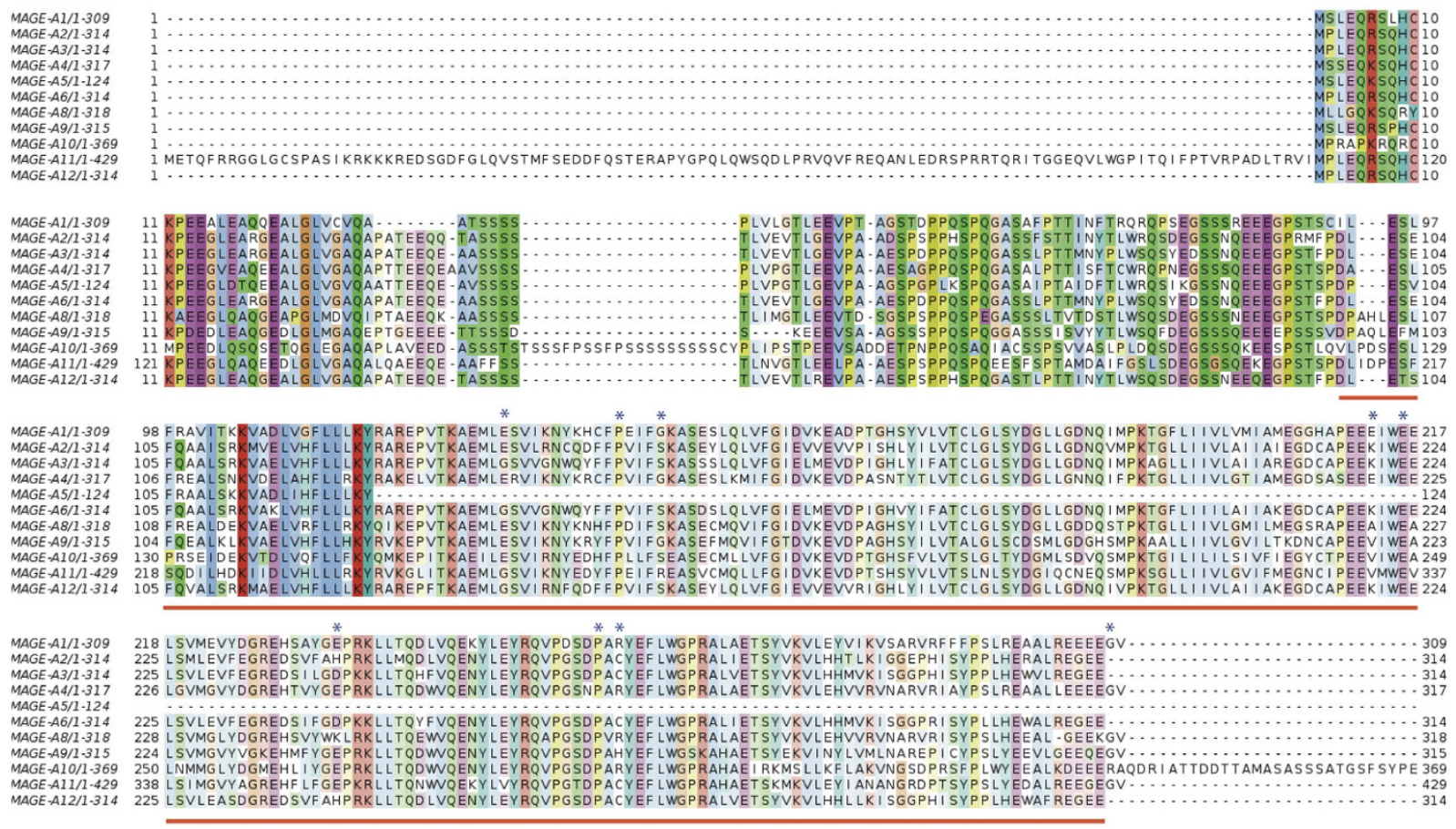

Figure 1. Multiple sequence alignment of MAGE-A proteins. Sequences are colored according to the Clustal coloring scheme. The MHD region is underlined in red. Residues that are mutated in this study are indicated with a star.

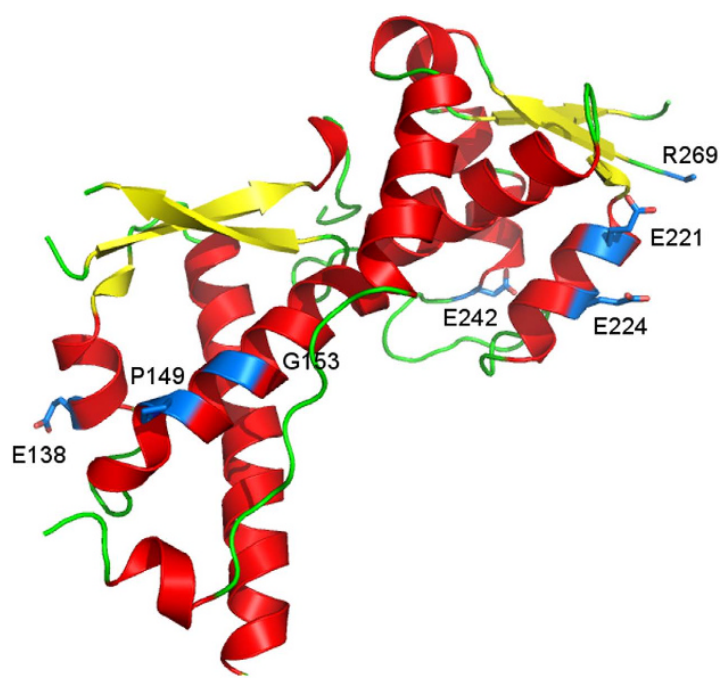

Figure 2. Crystal structure of MAGE-A4 MHD (PDB ID: 2WA0). The structure depicts the secondary structure helices in red and strands in yellow. The mutated residues that have been investigated in this study are shown in blue. Note that P267 and G316 are not visible in the structure. The figure was produced using Pymol.

Molecular modelling. Using Modeller ${ }^{30}$ we modelled the point mutations in the MAGE-A4 structure in order to understand the structural basis for the observed differences in the thermal stability. The residues Glu242 and Glu221 have the most pronounced effect on the stability. The Glu242 residue is located at the N-cap of helix 3 of the second winged helix domain. It H-bonds to the exposed backbone NH of the residues Arg244 and Lys245 in the first helical turn (Fig. 5a). This residue is fairly conserved amongst MAGE-A4 homologs suggesting the importance of this position. The mutation to Lys could cause a loss of the H-bonding network and this can account for the drastic loss of stability we have observed. The Glu221 residue is located on the second helix of the second winged helix domain. Mutation to Lys could allow the formation of a salt bridge between Lys221 and Glu213 and this can potentially increase the stability of the mutant E221K MAGE-A4 protein (Fig. 5b). The effect of the remaining mutations on the structure is less obvious. The Pro267 and Arg269 residues are located at 



Figure 3. Far-UV CD spectra of WT and mutant MAGE-A4 proteins. Spectra in the region of $260-195 \mathrm{~nm}$ were obtained at $6^{\circ} \mathrm{C}, 20^{\circ} \mathrm{C}, 37^{\circ} \mathrm{C}, 90^{\circ} \mathrm{C}, 37^{\circ} \mathrm{C}$ after heating to $90^{\circ} \mathrm{C}$, and at $20^{\circ} \mathrm{C}$ after heating to $90^{\circ} \mathrm{C}$. (a) WT, (b) E138K, (c) P149S, (d) G153D, (e) E221K, (f) E224K, (g) E242K, (h) P267S, (i) R269C and (j) G316R.

a loop region that is disordered in the crystal structure. Mutation of both residues may have a destabilizing conformational effect that can vary in magnitude depending on the residue location within the loop. The Pro149 and Gly153 residues are located on the third helix of the first winged helix domain. Both residues are probably important for the stability of the alpha-helix, particularly Pro149, as it is located at the N-terminal turn of the helix and is entropically favoured in this position. A substitution of Gly153 with a residue with side chain and charge such as Glu would be sterically unfavoured as it may clash with the side chain of the residue Ile 300 . The Glu138 residue 


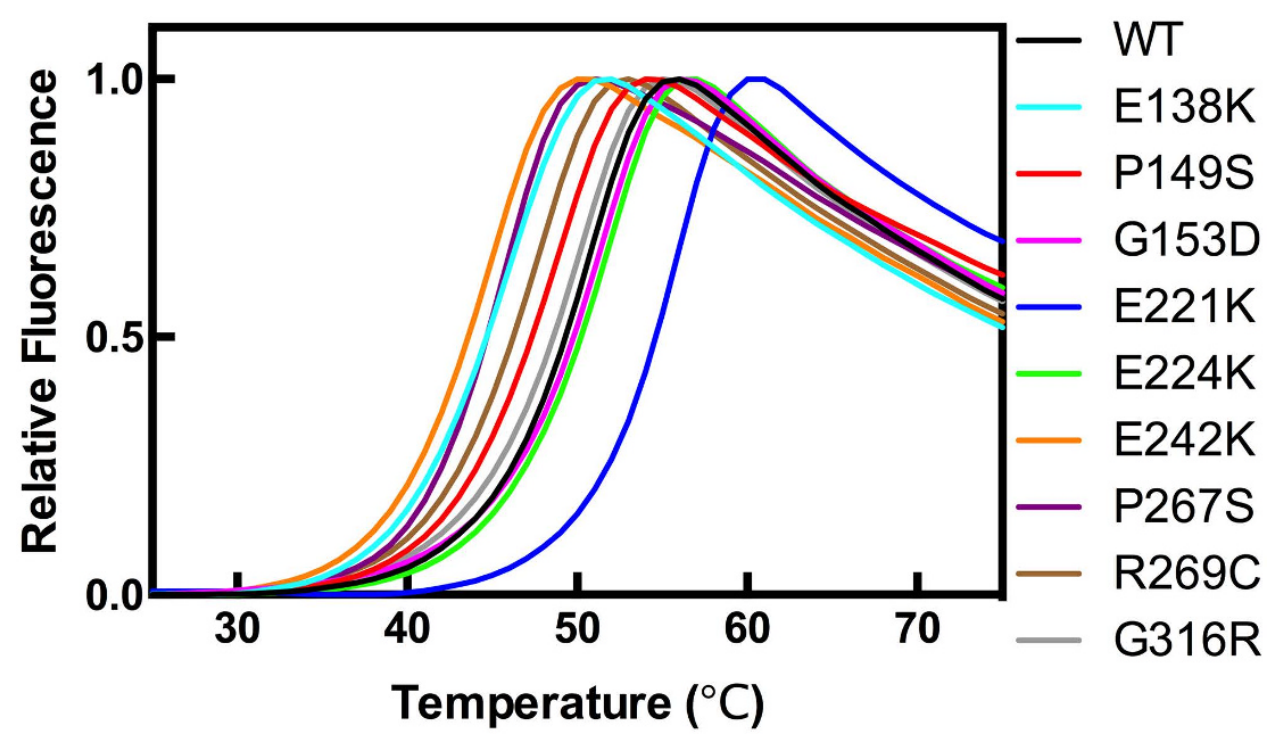

Figure 4. Thermal unfolding curves of MAGE-A4 and its cancer-associated mutants recorded by DSF. The data were recorded using MX3005P machine (Strategene) with the excitation and emission of SYPRO Orange dye set to be $470 \mathrm{~nm}$ and $570 \mathrm{~nm}$, respectively. The proteins were diluted to $2 \mu \mathrm{M}$ in $50 \mathrm{mM}$ Tris buffer containing $150 \mathrm{mM} \mathrm{NaCl}, 5 \mathrm{mM}$ DTT and 5\% glycerol, $\mathrm{pH} 7.2$.

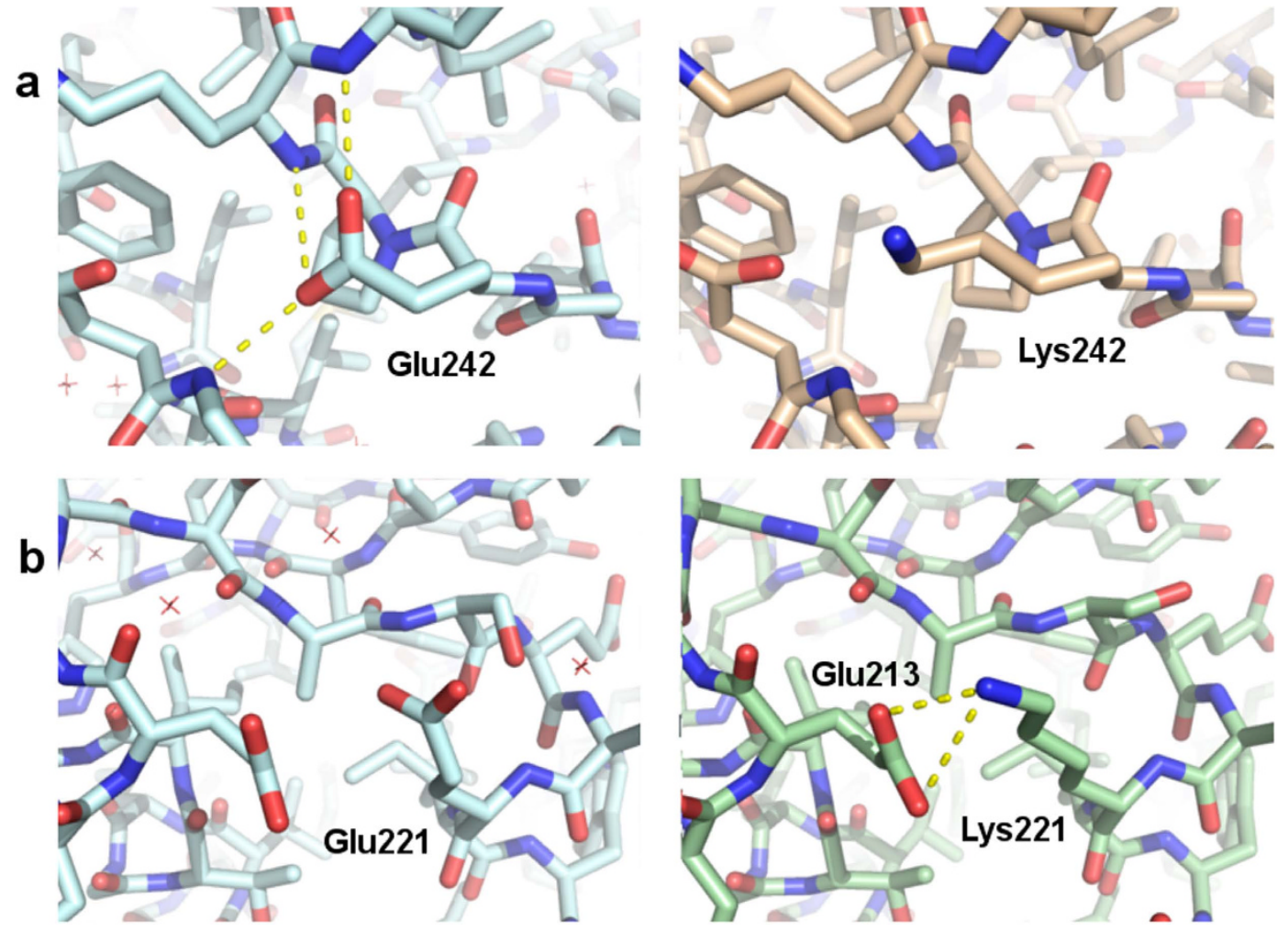

Figure 5. (a) Side by side comparison of the structure of wild type MAGE-A4 (PDB ID: 2WA0) and the theoretical model of the E242K mutant. (b) Side by side comparison of the structure of wild type MAGE-A4 (PDB ID: 2WA0) and the theoretical model of the E221K mutant.

is located at helix 2 of the first winged helix domain. Mutation of this residue to Lys may be unfavoured due to charge repulsion as this residue is in close vicinity to Lys142, Lys145 and Arg139.

1D proton NMR of MAGE-A4 proteins. 1D proton NMR was employed to examine the folding and conformation of MAGE-A4 protein and to assess the effects of the mutations in such properties. The spectra of the 


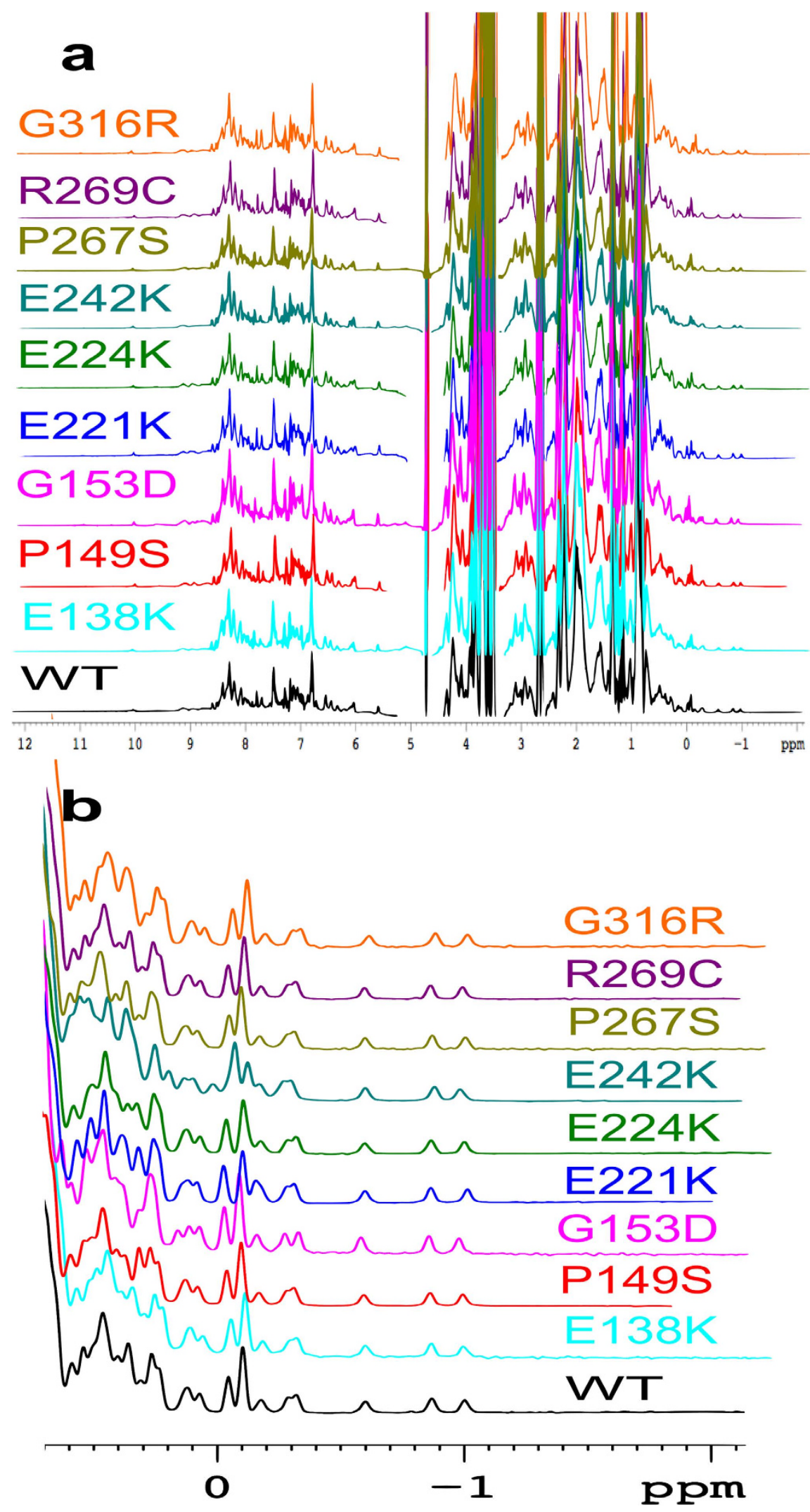

Figure 6. 1D Proton NMR Spectra of WT MAGE-A4 and its cancer-associated mutants. (a) Whole Spectra. (b) Zoomed-in spectra within 0.7 and $-2 \mathrm{ppm}$. Each protein was concentrated to $200 \mu \mathrm{M}$ in $50 \mathrm{mM}$ phosphate buffer ( $\mathrm{pH} \mathrm{7.2)} \mathrm{containing} 50 \mathrm{mM} \mathrm{NaCl}$ and $5 \mathrm{mM}$ DTT. The spectra were recorded at $600 \mathrm{MHz}, 25^{\circ} \mathrm{C}$. 


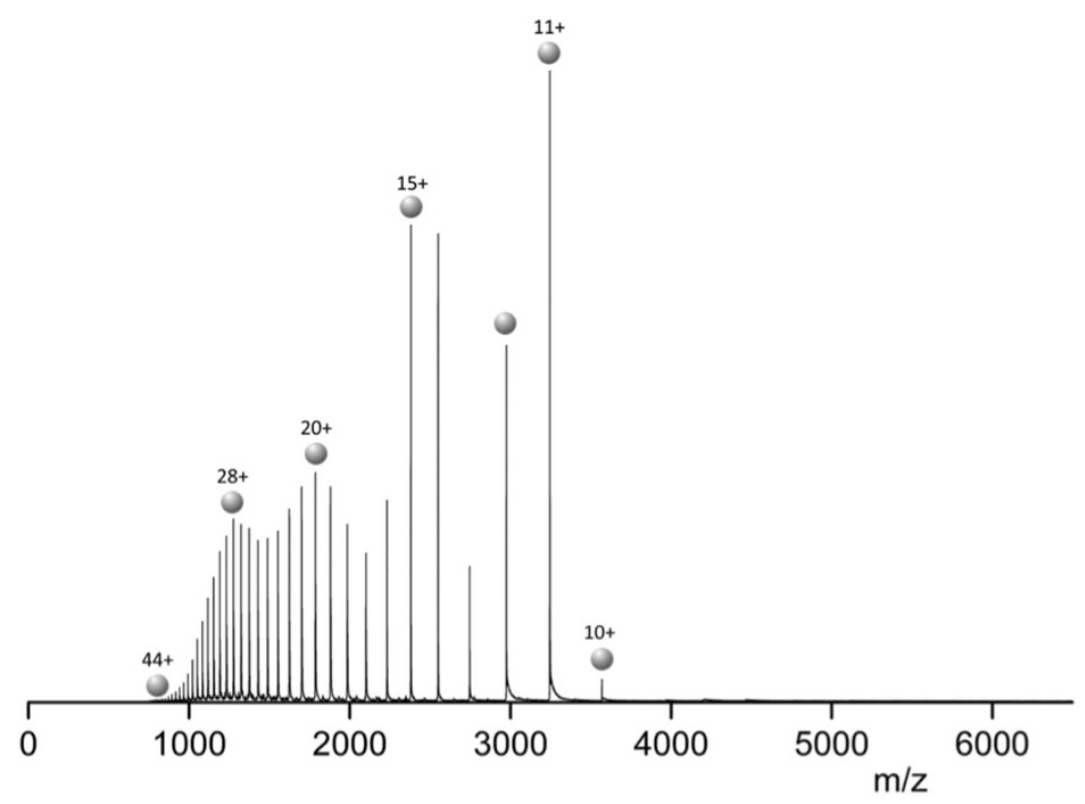

Figure 7. nESI mass spectrum for $20 \mu \mathrm{M}$ WT MAGE-A4. WT MAGE-A4 sprayed from aqueous solution containing $50 \mathrm{mM}$ Ammonium Acetate at $\mathrm{pH}$ 6.8. The protein exhibits a wide, multimodal, monomeric charge state range $10 \leq z \leq 44$. Several charge states are denoted with single spheres along with their respective charge.

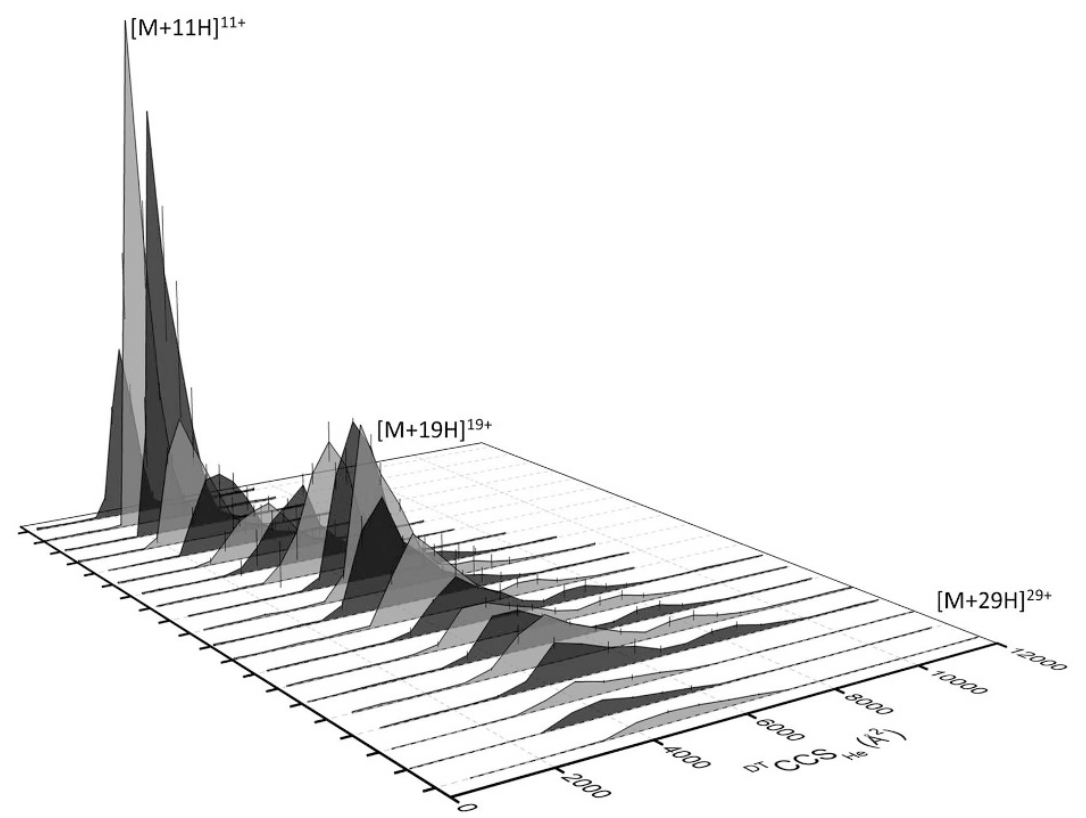

Figure 8. Collision cross section distributions (CCSDs) derived from arrival time distributions (ATDs) for WT MAGE-A4 at drift voltage $35 \mathrm{~V}$. The $\mathrm{x}$-, $\mathrm{y}$ - and $\mathrm{z}$-axes represent collision cross section (CCS, $\left.\AA^{2}\right)$, intensity directly acquired from IM-MS experiment, and charge state, respectively. Significant charge states have been labelled.

proteins are shown in Fig. 6a. The differences in the spectra between the WT and mutant proteins are minimal and located within the chemical shift of methyl protons (below $0.8 \mathrm{ppm}$ ). The NMR spectra for all of the tested MAGE-A4 proteins showed excellent dispersion of peaks between -1 and $0.8 \mathrm{ppm}$ that are typical of ring-current shifted protons located above or below the plane of aromatic side-chain. This indicates the presence of a structured hydrophobic core. However a large peak at $<1 \mathrm{ppm}$ was also observed for each protein, which is indicative of unstructured and disordered regions. These results suggest that the WT and mutant MAGE-A4 proteins contain a folded structure as well as disordered region(s). Figure $6 \mathrm{~b}$ zooms in the chemical shift region below $0.8 \mathrm{ppm}$ where the difference in the peaks is observed. While the spectra of P267S and R269C overlap completely with that 
of WT, the spectra of other mutants differ from that of WT in the chemical shift region from 0.7 to $-0.2 \mathrm{ppm}$. These small changes in the positions and patterns of the peaks reflect minor changes in the hydrophobic core caused by such mutations even though these mutations are located on the solvent-exposed surface of the protein. These mutations may somehow change the position or orientation of the planes of aromatic and/or methyl groups, changing the distances and/or angles of the interactions between methyl groups and aromatic planes.

MS and IM-MS of MAGE-A4. We employed MS and drift time IM-MS to probe the structure of WT and mutant MAGE-A4. The mass spectrum for WT MAGE-A4 is shown in Fig. 7. The protein presents in a wide charge state distribution (CSD) $10 \leq z \leq 44$. The most intense charge state is $[\mathrm{M}+11 \mathrm{H}]^{10+}$ although the distribution is multimodal and other intense species include $[\mathrm{M}+15 \mathrm{H}]^{15+},[\mathrm{M}+20 \mathrm{H}]^{20+}$ and $[\mathrm{M}+28 \mathrm{H}]^{28+}$. The CSD was easily malleable, changing significantly with ionic strength and cone potential. As the salt concentration increased the intensity of the higher charge states decreased somewhat, but they were still present over the same range (data not shown). The protein predominantly presents as a monomer of mass 35, 679 (cf. predicted mass of 35,684 Da) with very low abundance peaks assigned to a dimeric form (Supplementary Fig. S1). The wide CSD is typical of an intrinsically disordered protein, with many solvent accessible protonatable sites ${ }^{31}$. The intense $[\mathrm{M}+11 \mathrm{H}]^{11+}$ species indicates WT MAGE-A4 may possess some stable secondary structure, or at the very least a globular form which is built around the solved MHD structure.

IM-MS reveals that WT MAGE-A4 presents in multiple conformational families (Fig. 8). The low charge states $[\mathrm{M}+10 \mathrm{H}]^{10+},[\mathrm{M}+11 \mathrm{H}]^{11+}$ and $[\mathrm{M}+12 \mathrm{H}]^{12+}$ present in a single conformational family at collision cross section (CCS) $\sim 2400 \AA^{2}$, likely corresponding to a stable compact form of the protein. At intermediate charge states $13 \leq z \leq 20$ MAGE-A4 presents in at least two poorly resolved conformational families centered at $\sim 3200$ and $4500 \AA^{2}$. At high charge states $21 \leq z \leq 26$ the protein exhibits very wide collision cross section distributions (CCSDs) with multiple conformational families present ranging from $\sim 3500$ to $7500 \AA^{2}$, indicative of a disordered protein with large extended states. The highest charge states $27 \leq z \leq 29$ exhibit a single broad CCSD centered on $\sim 4000 \AA^{2}$, possibly due to gas-phase collapse. Charge states higher than $[\mathrm{M}+29 \mathrm{H}]^{29+}$ were not of sufficient intensity to obtain IM-MS data.

Mutant MAGE-A4 spectra are shown in Supplementary Fig. S2. All mutants present a wide CSD similar to that of WT MAGE-A4. For each case the proteins present as mainly monomer, exhibiting a multimodal distribution with the $[\mathrm{M}+11 \mathrm{H}]^{11+}$ species being the most intense. E138K MAGE-A4 presents in a monomeric charge state range $8 \leq z \leq 35$ with some low intensity low charge dimers. P149S MAGE-A4 presents in charge state range $8 \leq z \leq 33$, with a higher abundance of dimeric species compared with the WT protein. G153D MAGE-A4 presents as a charge state range $7 \leq z \leq 19$, with significantly fewer high charge states compared with the WT protein. As for WT MAGE-A4, G153D MAGE-A4 presents with very low abundance dimeric species. E221K MAGE-A4 presents in charge state range $7 \leq z \leq 21$ with some low intensity low charge dimeric species. E224K presents in charge state range $7 \leq z \leq 20$, again with the $[\mathrm{D}+15 \mathrm{H}]^{15+}$ and $[\mathrm{D}+17 \mathrm{H}]^{17+}$ dimeric species. E242K presents in a charge state range $7 \leq z \leq 18$ with low intensity $[\mathrm{D}+15 \mathrm{H}]^{15+}$ dimer. This charge state range is narrower, and dimer abundance is lower than the WT MAGE-A4 protein. P267S MAGE-A4 presents in a monomeric charge state range $7 \leq z \leq 22$. The dimeric species are low intensity with charge state range $15 \leq z \leq 39$, the highest abundance of dimeric species of WT and mutant MAGE-A4. R269C presents as a monomeric charge state range $7 \leq z \leq 17$, with $[\mathrm{D}+15 \mathrm{H}]^{15+}$ and $[\mathrm{D}+17 \mathrm{H}]^{17+}$ dimers. G316R presents with a monomeric charge state range $7 \leq z \leq 31$ and a dimeric charge state range $15 \leq z \leq 25$.

All mutants present with a narrower charge state range compared with the WT MAGE-A4, with G153D, E242K and R269C displaying a significantly narrower distribution, centered on lower charges suggestive of a more compact form being dominant in solution. MAGE-A4 mutants P149S, P267S and G316R display an increased abundance of dimeric species compared with the WT protein.

We performed IM-MS on the MAGE-A4 mutants which exhibited significant thermal stability differences; the most stable, E221K, and the least stable, E242K MAGE-A4 (Supplementary Fig. S3). E221K MAGE-A4 presents in similar conformational families to the WT protein for low charge states $9 \leq z \leq 12$, with a single conformational family centered at $2400 \AA^{2}$. At intermediate charge states $13 \leq z \leq 16$ E221K MAGE-A4 exhibits two conformational families centered at $\sim 2600$ and $\sim 4000 \AA^{2}$; similar to that of WT MAGE-A4 but with a narrower distribution. The high charge states $17 \leq z \leq 20$ present in much more narrow CCSDs for that observed in the WT protein. We see no highly extended conformational states, instead observing a broad unresolved distribution at $\sim 3500-5000 \AA^{2}$. The E242K MAGE-A4 protein again exhibits conformational differences in comparison with WT MAGE-A4. The trend of a single conformational family at low charge states, with multiple conformational families presenting at charge states $13 \leq z \leq 23$ remains consistent. We again however do not observe the more unfolded states present in the high charge states of WT MAGE-A4, and observe consistently much narrower CCSDs than both WT and E221K MAGE-A4. Several charge states are directly compared for the WT, E221K and E242K MAGE-A4 proteins (Supplementary Fig. S4), highlighting the similar CCSDs for the compact, low charge states but significant differences in the CCSD width at both intermediate and especially high charge states for the two mutant MAGE-A4 proteins. The mutations which impact on thermal stability, both positively and negatively, appear to also have an effect on the conformation landscape of MAGE-A4. The narrowing of the CCSDs suggests that E221K and E242K MAGE-A4 proteins possess less flexibility than WT MAGE-A4, preferentially presenting in more compact conformational states.

\section{Discussion}

Despite being discovered more than 20 years ago, biological functions of the MAGE family of proteins still remain poorly understood. Most studies were focused on developing anti-cancer immune therapy using the exclusiveness of type-I MAGE expression in cancer cells. For example, MAGE-A3 has been studied as a candidate for developing immune therapy ${ }^{32-34}$. Emerging data highlight their active roles in promoting cancer growth and 
malignance ${ }^{35-39}$. MAGE-A2 has been reported to repress the activity of tumor suppressor protein p53 through recruitment of HDAC or by inhibiting the interaction between 553 and DNA ${ }^{23,24}$.

In this study, we have selected MAGE-A4 and nine of its mutants identified in human cancer cells to obtain a first insight into their structural and biophysical properties. We observed that the selected point mutations have little effects on the structural integrity and folding of the protein probably since most of these mutated residues are located on solvent-exposed surface. However some mutations, such as E221K and E242K, had significant effects on the thermal stability. The apparent $T_{\mathrm{m}}$ of E242K MAGE-A4 was $5.7^{\circ} \mathrm{C}$ lower than that of WT protein. A small fraction ( $c a 10 \%)$ of this MAGE-A4 mutant unfolds at physiological temperature. The decreased thermal stability coupled with unfolding at physiological temperature caused by E242K mutation may reduce the functional activities of MAGE-A4 protein in vivo.

Both NMR and native MS showed for the first time that these MAGE-A4 proteins contain a compact folded structure as well as disordered region(s). Most of such disordered regions are expected to be located within the $\mathrm{N}$-terminal 100 residues due to high presence of disorder promoting residues. Interestingly, we observed a very small fraction of dimeric species in the native MS. It has been reported that MAGE-A11 can form dimer ${ }^{40}$. In addition, while this article was under review, a new report emerged suggesting that MAGE-A3 presents predominantly as dimer in solution ${ }^{41}$. It is not known whether such dimeric species possess different functional properties compared to the monomers.

Of note, the percentage of MAGE-A4-positive patient tumors observed in select cancer subtypes has been reported as follows: $47 \%$ in ovarian cancers, $19-35 \%$ in lung cancers, $22 \%$ in colon cancers and $13 \%$ in breast cancers $^{39}$. The role of MAGE-A4 in cancers is not fully understood especially at macromolecular level. In fact, there are conflicting reports regarding its role in cancer. It has been reported to have oncogenic properties by inhibiting p53 downstream genes such as $B A X$ and $p 21$, and thus promoting cell growth in normal oral keratinocytes ${ }^{22}$. However MAGE-A4 was also reported to have tumor suppressive functions as it was shown to promote apoptosis in non-small cell lung cancers ${ }^{42}$. It is not understood how MAGE-A4 exerts such opposing roles. We hypothesize that such observations may arise from different interacting partners of MAGE-A4. As part of its tumor repressing mechanism, MAGE-A4 has been indicated to bind with gankyrin, suppressing its oncogenic activity ${ }^{26}$. However, to date, no direct molecular interactions have been identified for MAGE-A4, which can lead to cancer growth.

As the point mutations selected for this study are located on the solvent exposed surface of MAGE-A4, even though they do not have effects on structural integrity of MHD as observed in this study, they may have a significant impact on the protein-protein interaction(s), which may enhance or repress tumor growth. In addition, conformational changes have been shown for E221K and E242K mutants in IM-MS experiments. These may also have effects on the protein-protein interaction(s), by modifying the conformational flexibility of MAGE-A4. Furthermore, the impact of the mutations on the thermal stability and folding of the protein may have significant effects on the functions of this protein in vivo. Further investigation needs to be conducted to identify other proteins that interact with MAGE-A4 and assess the impact of these mutations on such interactions to understand whether these mutations have different functional roles in cancer.

Given the significance of MAGE-A4 in biology and specifically in cancer, it is important to fully understand the functions of this protein at the molecular level in order to exploit it in translational research. Specifically, the mutations may affect the interactions with key binding cell cycle proteins. The latter are subject to further investigations aiming to identify and characterise such binding proteins in the hope to better understand the role of these mutations in cancer.

\section{Methods}

MAGE-A4 cloning and site-directed mutagenesis. The DNA of WT MAGE-A4 was cloned into pGEX-6p-1 (GE Healthcare) to express GST-tagged MAGEA-4 using primer-1: 5'-AAAAAAGAATTCATGTCTTCTGAGCAGA AGAGTCAG-3' as a forward primer, primer-2: AAAAAACTCGAGTCAGACTCCCTCTTCCTCTAA-3' as a reverse primer, and vector $\mathrm{pGEX}-4 \mathrm{~T}-1$ containing MAGE-A4 DNA sequence ${ }^{43}$ as a template. MAGE-A4 mutants were generated using QuickChange ${ }^{\circledR}$ Lightning Site-Directed Mutagenesis Kit (Agilent Technologies).

Protein expression and purification. Proteins were expressed in Escherichia coli C41 (DE3) cells. Single colonies were inoculated into $5 \mathrm{ml} \mathrm{LB}$ media (ForMedium) supplemented with $100 \mu \mathrm{g} / \mathrm{ml}$ ampicillin, followed by incubation at $37^{\circ} \mathrm{C}$ overnight. $5 \mathrm{ml}$ of the overnight culture was added into $500 \mathrm{ml} \mathrm{LB}$ with $100 \mu \mathrm{g} / \mathrm{ml}$ ampicillin, followed by incubation at $37^{\circ} \mathrm{C}$ until the $\mathrm{OD}_{600}$ reached 0.6 . The culture was then allowed to cool down to $21^{\circ} \mathrm{C}$ and the protein expression was induced with $1 \mathrm{mM}$ isopropyl $\beta$-D-thiogalactoside overnight. The cultures were centrifuged at $11300 \times \mathrm{g}, 4^{\circ} \mathrm{C}$ for $20 \mathrm{~min}$. The cells were collected and lysed using $5 \mathrm{ml} / \mathrm{g}$ cell of BugBuster ${ }^{\circledR}$ protein extraction reagent (Novagen) containing 1 tablet of complete ${ }^{\circledR}$ EDTA- free protease inhibitor (Roche), 125 $\mathrm{U}$ of Benzonase nuclease (Novagen) and $5 \mathrm{mM}$ dithiothreitol (DTT). The cell lysate was centrifuged at $20000 \times \mathrm{g}$, $4^{\circ} \mathrm{C}$ for $40 \mathrm{~min}$. The soluble fraction was collected and the protein was purified using GST GraviTrap columns (GE Healthcare). The GST-tagged protein was incubated with $5 \mathrm{U} / \mathrm{mg}$ protein of PreScission protease (GE Healthcare) at $4{ }^{\circ} \mathrm{C}$ overnight. The protein was passed again through GST GraviTrap column to remove the GST, followed by further purification using $26 / 60$ Superdex 200 column connected to ÄKTAprime ${ }^{\circledR}$ system equipped with Prime View software (GE Healthcare). The purified protein was concentrated using Amicon Ultra-15 centrifugal filter unit, 10,000 NMWL (Merck Millipore) and stored at $-80^{\circ} \mathrm{C}$.

CD. Simultaneous UV absorption and CD spectra of MAGE4 proteins were acquired on the Chirascan Plus ${ }^{\circledR}$ spectrometer, equipped with a Quantum Northwest TC125 Peltier unit (Applied Photophysics, Leatherhead, UK). The instrument was flushed continuously with pure evaporated nitrogen gas throughout the measurements. Far-UV CD spectra were recorded with a $2.0 \mathrm{~nm}$ spectral bandwith, a $1.0 \mathrm{~nm}$ step size and a $1.5 \mathrm{~s}$ instrument 
time-per-point. A $0.5 \mathrm{~mm}$ Suprasil rectangular cell (Hellma UK Ltd) was employed in the region 260-195 nm. Far-UV CD spectra were recorded at room temperature $\left(20^{\circ} \mathrm{C}\right)$, cooled to $6^{\circ} \mathrm{C}$, then heated to high temperatures $\left(37^{\circ} \mathrm{C}\right.$ and $\left.90^{\circ} \mathrm{C}\right)$ and re-cooled to $37^{\circ} \mathrm{C}$ and $20^{\circ} \mathrm{C}$. The temperature was measured directly with a thermocouple probe in the sample solution. Protein samples were concentrated to $0.2 \mathrm{mg} / \mathrm{ml}$ and were buffer exchanged to $20 \mathrm{mM}$ Tris buffer containing $50 \mathrm{mM} \mathrm{NaCl}$ and $1 \mathrm{mM}$ dithioerythritol (DTE), pH 7.2. All CD spectra were buffer baseline subtracted and then corrected for concentration and path length and expressed in terms of $\Delta \varepsilon$ $\left(\mathrm{M}^{-1} \mathrm{~cm}^{-1}\right)$ per amino acid residue $(\mathrm{MW}=113)$. Protein secondary structure prediction was performed using K2D3 (http://k2d3.ogic.ca/) ${ }^{29}$.

DSF. Protein samples were diluted to $2 \mu \mathrm{M}$ in $50 \mathrm{mM}$ Tris buffer containing $150 \mathrm{mM} \mathrm{NaCl}, 5 \mathrm{mM}$ DTT, and $5 \%$ glycerol. SYPRO ${ }^{\circledR}$ Orange stain (Sigma-Aldrich) was added into the samples to be diluted 500 times. $20 \mu \mathrm{l}$ of each sample was transferred to 96-well plate, and the plate was sealed with a transparent tape. The samples were run in MX3005P machine (Strategene) connected to MxPro software and the temperature was increased from $25^{\circ} \mathrm{C}$ to $95^{\circ} \mathrm{C}$. The stain was excited at $470 \mathrm{~nm}$ and the emission at $570 \mathrm{~nm}$ was measured. The unfolding curve was generated by normalizing the fluorescence values using equation:

$$
\frac{\mathrm{F}_{\mathrm{T}}-\mathrm{F}_{\text {Min }}}{\mathrm{F}_{\text {Max }}-\mathrm{F}_{\text {Min }}}
$$

where $\mathrm{F}_{\mathrm{T}}$ is fluorescence at temperature $\mathrm{T}, \mathrm{F}_{\mathrm{Max}}$ is maximum fluorescence, and $\mathrm{F}_{\mathrm{Min}}$ is minimum fluorescence.

Molecular modelling. Models were built with Modeller ${ }^{30}$ using the structure of human MAGEA4 as template (PDB ID: 2WA0).

1D proton NMR. Protein samples were concentrated to $200 \mu \mathrm{M}$ and the buffer was exchanged to $50 \mathrm{mM}$ phosphate sodium buffer containing $50 \mathrm{mM} \mathrm{NaCl}$ and $5 \mathrm{mM}$ DTT. $500 \mu \mathrm{l}$ of each protein sample was mixed with $50 \mu \mathrm{l}$ of deuterium oxide and transferred to an NMR tube. The proton NMR spectra were recorded using Bruker Avance III 600 at $25^{\circ} \mathrm{C}, 600 \mathrm{MHz}$.

MS. Prior to analysis, samples were thawed and dialyzed using Bio-RAD micro bio-spin chromatography columns (Bio-Rad Laboratories, Inc) into $50 \mathrm{mM}$ Ammonium Acetate and diluted to $20 \mu \mathrm{M}$. Protein concentrations were confirmed post dialysis spectrophotometrically (NanoDrop Spectrophotometer ND 1000, Thermo Scientific, USA). Mass Spectrometry experiments were performed on a Micro Mass Quadruple Time-of-Flight Ultima Global (Waters, Manchester, UK) and ion mobility mass spectrometry measurements were made on an in-house modified quadrupole time-of-flight mass spectrometer including a $5.1 \mathrm{~cm} \mathrm{drift} \mathrm{cell}{ }^{44}$. Ions were produced by positive nano-electrospray ionisation (nESI), with a spray voltage range of $1.55-1.69 \mathrm{kV}$ and a source temperature of $80^{\circ} \mathrm{C}$. nESI glass capillaries were prepared in-house from thin-walled borosilicate capillaries (Precision Instruments, Stevenage, UK) using a Flaming/Brown micropipette puller Model P-97 (Sutter Instrument company, Novato, CA, USA). The IM-MS drift cell was filled with Helium gas, to pressure of 3.56-3.75 Torr, measured using a baratron (MKS Instruments). The electric potential of the drift cell was varied from 12 to $2 \mathrm{Vcm}^{-1}$. Ion arrival time distributions (ATDs) were recorded by synchronisation of the release of ions into the drift cell with mass spectral acquisition. ATDs were converted into collision cross section distributions using equation:

$$
\Omega_{\mathrm{avg}}=\frac{(18 \pi)^{1 / 2}}{16}\left[\frac{1}{m_{b}}+\frac{1}{m}\right]^{1 / 2} \frac{z e}{\left(k_{B} T\right)^{1 / 2}} \frac{1}{\rho} \frac{t_{D} V}{L^{2}}
$$

where $\Omega$ is the rotationally averaged collision cross section $\left(\AA^{2}\right), \mathrm{m}$ and $\mathrm{m}_{\mathrm{b}}$ are the masses of the ion and the buffer gas, $z$ is the ion charge, $e$ is the elementary charge, $\mathrm{k}_{\mathrm{B}}$ is the Boltzmann constant, $\rho$ is the buffer gas density, $\mathrm{T}$ is the gas temperature, $\mathrm{L}$ is the drift cell length, $\mathrm{V}$ is the voltage applied across the drift cell (here $35 \mathrm{~V}$ ) and $t_{D}$ is the drift time. The arrival time of the ions $\left(t_{a}\right)$ includes the time spent within the mass spectrometer by outside of the drift cell, also known as the dead time $\left(t_{0}\right)$. The value for $t_{0}$ is calculated by taking an average value of the intercept from a linear plot of average arrival time versus pressure/temperature and was subtracted from the arrival time to calculate drift time $\left(t_{D}\right)$ :

$$
\mathrm{t}_{\mathrm{D}}=\mathrm{t}_{\mathrm{a}}-\mathrm{t}_{0}
$$

All processing was carried out using Mass Lynx V4.1 (Waters Corporation) and Origin 9.0 (Origin Lab Corporation, USA) software. Intensities of the CCSD peaks was directly taken from peak intensity during IM-MS experiments for three analytical repeats, in Mass Lynx V4.1 software (Waters Corporation).

\section{References}

1. Türeci, O. et al. Expression of SSX genes in human tumors. Int. J. Cancer 77, 19-23 (1998)

2. Sahin, U. et al. Expression of Cancer Testis Genes in Human Brain Tumors. Clin. Cancer Res. 6, 3916-3922 (2000).

3. Scanlan, M. J. et al. Expression of cancer-testis antigens in lung cancer: definition of bromodomain testis-specific gene (BRDT) as a new CT gene, CT9. Cancer Lett. 150, 155-164 (2000).

4. Mashino, K. et al. Expression of multiple cancer-testis antigen genes in gastrointestinal and breast carcinomas. Br. J. Cancer $\mathbf{8 5}$, 713-720 (2001)

5. Scanlan, M. J., Gure, A. O., Jungbluth, A. A., Old, L. J. \& Chen, Y.-T. Cancer/testis antigens: an expanding family of targets for cancer immunotherapy. Immunol. Rev. 188, 22-32 (2002). 
6. Knuth, A., Wölfel, T., Klehmann, E., Boon, T. \& Meyer zum Büschenfelde, K. H. Cytolytic T-cell clones against an autologous human melanoma: specificity study and definition of three antigens by immunoselection. Proc. Natl. Acad. Sci. USA 86, 2804-2808 (1989).

7. van der Bruggen, P. et al. A gene encoding an antigen recognized by cytolytic T lymphocytes on a human melanoma. Science 254, 1643-1647 (1991).

8. Traversari, C. et al. Transfection and expression of a gene coding for a human melanoma antigen recognized by autologous cytolytic T lymphocytes. Immunogenetics 35, 145-152 (1992).

9. De Plaen, E. et al. Structure, chromosomal localization, and expression of 12 genes of the MAGE family. Immunogenetics 40, 360-369 (1994).

10. Rogner, U. C., Wilke, K., Steck, E., Korn, B. \& Poustka, A. The melanoma antigen gene (MAGE) family is clustered in the chromosomal band Xq28. Genomics 29, 725-731 (1995).

11. Dabovic, B. et al. A family of rapidly evolving genes from the sex reversal critical region in Xp21. Mamm. Genome 6, 571-580 (1995).

12. Muscatelli, F., Walker, A. P., De Plaen, E., Stafford, A. N. \& Monaco, A. P. Isolation and characterization of a MAGE gene family in the Xp21.3 region. Proc. Natl. Acad. Sci. 92, 4987-4991 (1995).

13. Lurquin, C. et al. Two members of the human MAGEB gene family located in Xp21.3 are expressed in tumors of various histological origins. Genomics 46, 397-408 (1997).

14. Lucas, S. et al. Identification of a New MAGE Gene with Tumor-specific Expression by Representational Difference Analysis. Cancer Res. 58, 743-752 (1998).

15. Lucas, S., Brasseur, F. \& Boon, T. A New MAGE Gene with Ubiquitous Expression Does Not Code for Known MAGE Antigens Recognized by T Cells. Cancer Res. 59, 4100-4103 (1999).

16. Põld, M. et al. Identification of a new, unorthodox member of the MAGE gene family. Genomics 59, 161-167 (1999).

17. Lucas, S., De Plaen, E. \& Boon, T. MAGE-B5, MAGE-B6, MAGE-C2, and MAGE-C3: four new members of the MAGE family with tumor-specific expression. Int. J. Cancer 87, 55-60 (2000).

18. Stone, B. et al. MAGE-F1, a novel ubiquitously expressed member of the MAGE superfamily. Gene 267, 173-182 (2001).

19. Barker, P. A. \& Salehi, A. The MAGE proteins: emerging roles in cell cycle progression, apoptosis, and neurogenetic disease. J. Neurosci. Res. 67, 705-712 (2002).

20. Doyle, J. M., Gao, J., Wang, J., Yang, M. \& Potts, P. R. MAGE-RING protein complexes comprise a family of E3 ubiquitin ligases. Mol. Cell 39, 963-974 (2010)

21. Glazer, C. A. et al. The role of MAGEA2 in head and neck cancer. Arch. Otolaryngol. Head. Neck Surg. 137, 286-293 (2011).

22. Bhan, S., Chuang, A., Negi, S. S., Glazer, C. A. \& Califano, J. A. MAGEA4 induces growth in normal oral keratinocytes by inhibiting growth arrest and apoptosis. Oncol. Rep. 28, 1498-1502 (2012).

23. Monte, M. et al. MAGE-A tumor antigens target $\mathrm{p} 53$ transactivation function through histone deacetylase recruitment and confer resistance to chemotherapeutic agents. Proc. Natl. Acad. Sci. USA 103, 11160-11165 (2006).

24. Marcar, L., Maclaine, N. J., Hupp, T. R. \& Meek, D. W. Mage-A cancer/testis antigens inhibit p53 function by blocking its interaction with chromatin. Cancer Res. 70, 10362-10370 (2010).

25. Wong, P.-P. et al. Identification of MAGEA antigens as causal players in the development of tamoxifen-resistant breast cancer. Oncogene 33, 4579-4588 (2014).

26. Nagao, T. et al. MAGE-A4 interacts with the liver oncoprotein gankyrin and suppresses its tumorigenic activity. J. Biol. Chem. 278, 10668-10674 (2003).

27. Sakurai, T. et al. A cleaved form of MAGE-A4 binds to Miz-1 and induces apoptosis in human cells. J. Biol. Chem. 279, 15505-15514 (2004).

28. Caballero, O. L. et al. Frequent MAGE mutations in human melanoma. Plos One 5, 7 (2010).

29. Louis-Jeune, C., Andrade-Navarro, M. A. \& Perez-Iratxeta, C. Prediction of protein secondary structure from circular dichroism using theoretically derived spectra. Proteins 80, 374-381 (2012).

30. Sali, A. \& Blundell, T. L. Comparative protein modelling by satisfaction of spatial restraints. J. Mol. Biol. 234, 779-815 (1993).

31. Beveridge, R. et al. A mass-spectrometry-based framework to define the extent of disorder in proteins. Anal. Chem. 86, 10979-10991 (2014).

32. Kruit, W. H. J. et al. Selection of immunostimulant AS15 for active immunization with MAGE-A3 protein: results of a randomized phase II study of the European Organisation for Research and Treatment of Cancer Melanoma Group in Metastatic Melanoma. J. Clin. Oncol. 31, 2413-2420 (2013).

33. Vansteenkiste, J. et al. Adjuvant MAGE-A3 immunotherapy in resected non-small-cell lung cancer: phase II randomized study results. J. Clin. Oncol. 31, 2396-2403 (2013).

34. Gérard, C., Baudson, N., Ory, T. \& Louahed, J. Tumor mouse model confirms MAGE-A3 cancer immunotherapeutic as an efficient inducer of long-lasting anti-tumoral responses. Plos One 9, e94883 (2014).

35. Jung, E. J. et al. Expression of family A melanoma antigen in human gastric carcinoma. Anticancer Res. 25, 2105-2111 (2005).

36. Bergeron, A. et al. High frequency of MAGE-A4 and MAGE-A9 expression in high-risk bladder cancer. Int. J. Cancer 125, 1365-1371 (2009).

37. Glazer, C. A. et al. The role of MAGEA2 in head and neck cancer. Arch. Otolaryngol. Head. Neck Surg. 137, 286-293 (2011).

38. Lian, Y. et al. Expressions of MAGE-A10 and MAGE-A11 in breast cancers and their prognostic significance: a retrospective clinical study. J. Cancer Res. Clin. Oncol. 138, 519-527 (2012).

39. Weon, J. L. \& Potts, P. R. The MAGE protein family and cancer. Curr. Opin. Cell Biol. 37, 1-8 (2015).

40. Bai, S. \& Wilson, E. M. Epidermal-growth-factor-dependent phosphorylation and ubiquitinylation of MAGE-11 regulates its interaction with the androgen receptor. Mol. Cell. Biol. 28, 1947-1963 (2008).

41. Newman, J. A. et al. Structures of Two Melanoma-Associated Antigens Suggest Allosteric Regulation of Effector Binding. Plos One 11, e0148762 (2016).

42. Peikert, T., Specks, U., Farver, C., Erzurum, S. C. \& Comhair, S. A. A. Melanoma antigen A4 is expressed in non-small cell lung cancers and promotes apoptosis. Cancer Res. 66, 4693-4700 (2006)

43. Peche, L. Y., Scolz, M., Ladelfa, M. F., Monte, M. \& Schneider, C. MageA2 restrains cellular senescence by targeting the function of PMLIV/p53 axis at the PML-NBs. Cell Death Differ. 19, 926-936 (2012).

44. McCullough, B. J. et al. Development of an ion mobility quadrupole time of flight mass spectrometer. Anal. Chem. 80, 6336-6344 (2008).

\section{Acknowledgements}

We thank Dr. Claudio Schneider for the MAGE-A4 clone. We wish to acknowledge the support of professor Brian Sutton during the course of this study.

\section{Author Contributions}

Y.H., F.H. and A.J. purified proteins, Y.H. and T.B. performed CD experiments, Y.H. and E.C. performed NMR and DSF and analyzed data, A.A. performed structure-based molecular modelling, E.R.D. and P.E.B. performed MS experiments and analyzed data. P.V.N. and Y.H. participated in project design, Y.H., F.H., T.T.T.B., L.S., E.R.D., E.C., A.A., P.E.B. and P.V.N. analyzed data and participated in the preparation of the manuscript. P.V.N. conceived and supervised the project. All authors read and provided feedback on the manuscript. 


\section{Additional Information}

Supplementary information accompanies this paper at http://www.nature.com/srep

Competing financial interests: The authors declare no competing financial interests.

How to cite this article: Hagiwara, Y. et al. Consequences of point mutations in melanoma-associated antigen 4 (MAGE-A4) protein: Insights from structural and biophysical studies. Sci. Rep. 6, 25182; doi: 10.1038/srep25182 (2016).

\section{(c) (1)}

This work is licensed under a Creative Commons Attribution 4.0 International License. The images or other third party material in this article are included in the article's Creative Commons license, unless indicated otherwise in the credit line; if the material is not included under the Creative Commons license, users will need to obtain permission from the license holder to reproduce the material. To view a copy of this license, visit http://creativecommons.org/licenses/by/4.0/ 